\title{
Perfect matching and distance spectral radius in graphs and bipartite graphs
}

\author{
Yuke Zhang and Huiqiu Lin* \\ Department of Mathematics, East China University of Science and Technology, \\ Shanghai 200237, P.R. China
}

\begin{abstract}
A perfect matching in a graph $G$ is a set of nonadjacent edges covering every vertex of $G$. Motivated by recent progress on the relations between the eigenvalues and the matching number of a graph, in this paper, we aim to present a distance spectral radius condition to guarantee the existence of a perfect matching. Let $G$ be an $n$-vertex connected graph where $n$ is even and $\lambda_{1}(D(G))$ be the distance spectral radius of $G$. Then the following statements are true.

I) If $4 \leq n \leq 10$ and $\lambda_{1}(D(G)) \leq \lambda_{1}\left(D\left(S_{n, \frac{n}{2}-1}\right)\right)$, then $G$ contains a perfect matching unless $G \cong S_{n, \frac{n}{2}-1}$ where $S_{n, \frac{n}{2}-1} \cong K_{\frac{n}{2}-1} \vee\left(\frac{n}{2}+1\right) K_{1}$.

II) If $n \geq 12$ and $\lambda_{1}(D(G)) \leq \lambda_{1}\left(D\left(G^{*}\right)\right)$, then $G$ contains a perfect matching unless $G \cong G^{*}$ where $G^{*} \cong K_{1} \vee\left(K_{n-3} \cup 2 K_{1}\right)$.

Moreover, if $G$ is a connected $2 n$-vertex balanced bipartite graph with $\lambda_{1}(D(G)) \leq$ $\lambda_{1}\left(D\left(B_{n-1, n-2}\right)\right)$, then $G$ contains a perfect matching, unless $G \cong B_{n-1, n-2}$ where $B_{n-1, n-2}$ is obtained from $K_{n, n-2}$ by attaching two pendent vertices to a vertex in the $n$-vertex part.
\end{abstract}

Keywords: Distance spectral radius; Perfect matching;

2000 Mathematics Subject Classification: 05C50

\section{Introduction}

All graphs considered in this paper are undirected, connected and simple. Let $G$ be a graph with vertex set $V(G)=\left\{v_{1}, v_{2}, \ldots, v_{n}\right\}$ and edge set $E(G)$. The distance between $v_{i}$ and $v_{j}$, denoted by $d_{G}\left(v_{i}, v_{j}\right)$ (or $d_{i j}$ ), is the length of a shortest path from $v_{i}$ to $v_{j}$. The distance matrix of $G$, denoted by $D(G)$, is the $n \times n$ real symmetric matrix whose $(i, j)$-entry is $d_{G}\left(v_{i}, v_{j}\right)$ (or $\left.d_{i j}\right)$, then we can order the eigenvalues of $D(G)$ as

$$
\lambda_{1}(D(G)) \geq \lambda_{2}(D(G)) \geq \cdots \geq \lambda_{n}(D(G)) .
$$

By the Perron-Frobenius theorem, $\lambda_{1}(D(G))$ is always positive (unless $G$ is trivial) and $\lambda_{1}(D(G)) \geq\left|\lambda_{i}(D(G))\right|$ for $i=2,3, \ldots, n$, and we call $\lambda_{1}(D(G))$ the distance spectral radius.

The study of distance eigenvalues can be traced back to 1971 by Graham and Pollack [6] and they described a relationship between the number of negative distance eigenvalues and

\footnotetext{
*Supported by the National Natural Science Foundation of China (Nos. 11771141 and 12011530064). E-mail: huiqiulin@126.com (H.Q. Lin), zhang_yk1029@163.com (Y.K. Zhang)
} 
the addressing problem in data communication system. In the same paper, they showed a very interesting and insightful result that the determinant of the distance matrix of a tree with order $n$ is $(-1)^{n-1}(n-1) 2^{n-2}$, which is independent of the structure of the tree. Since then, the study of distance eigenvalues of a graph has become a research subject of enormous interest and this topic has received growing attention over recent years, some latest results see [1, 10, 11, 13, 17]. For more results on the distance matrix and its spectral properties, we refer the reader to the excellent survey [2].

A matching $M$ of a graph $G$ is a set of pairwise nonadjacent edges. The maximum number of edges of a matching in $G$ is called matching number of $G$, denoted by $\alpha(G)$. A vertex incident with an edge in $M$ is called to be saturated by $M$. A perfect matching is one matching which all vertices of $G$ are saturated by it. Obviously, a graph with a perfect matching has an even number of vertices and $\alpha(G)=\frac{|V(G)|}{2}$.

The studies of the connections between eigenvalues and the matching number of a graph were mainly based on the tree in 1990s. Chang [4] obtained an upper bound and a tight lower bound for the second largest eigenvalue of an $n$-vertex tree with a given matching number. Later, Hou and Li [8] gave some upper bounds for the spectral radius of a tree in terms of its order and matching number. Brouwer and Heamers [3] investigated this problem to the general graph and showed that if $\mu_{1}(G) \leq 2 \mu_{n-1}(G)$, then $G$ contains a perfect matching where $\mu_{1}(G)$ and $\mu_{n-1}(G)$ are the largest and second smallest Laplacian eigenvalues of $G$, respectively. Feng, Yu and Zhang [5] investigated the maximal spectral radius of graphs with a given matching number and order. In the past decade, some quality and interesting results on the matching number of a graph and its distance spectral radius have been obtained. Ilić [9] characterized $n$-vertex trees with a given matching number which minimize the distance spectral radius. Liu [12] characterized graphs with minimum distance spectral radius in connected graphs on $n$ vertices with fixed matching number. Zhang [19] and Lu and Luo [14] characterized unicyclic graphs with a perfect matching and a given matching number which minimize the distance spectral radius, respectively.

Very recently, O [15] proved a lower bound for the spectral radius in an $n$-vertex graph to guarantee the existence of a perfect matching. Along this line, we consider this problem with respect to the distance spectral radius in this paper.

We denote by $G \cup H$ the disjoint union of two graphs $G$ and $H$, which is the graph with $V(G \cup H)=V(G) \cup V(H)$ and $E(G \cup H)=E(G) \cup E(H)$, particularly if $G \cong H$, 


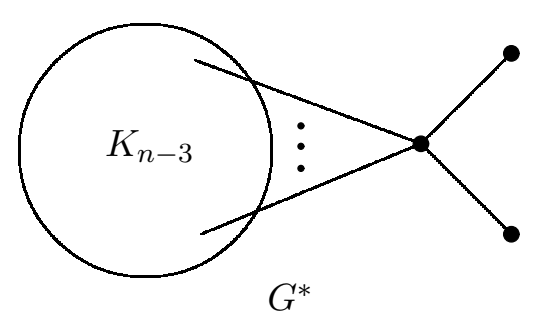

Figure 1: The extremal graph $G^{*}$ of Theorem 1.1.

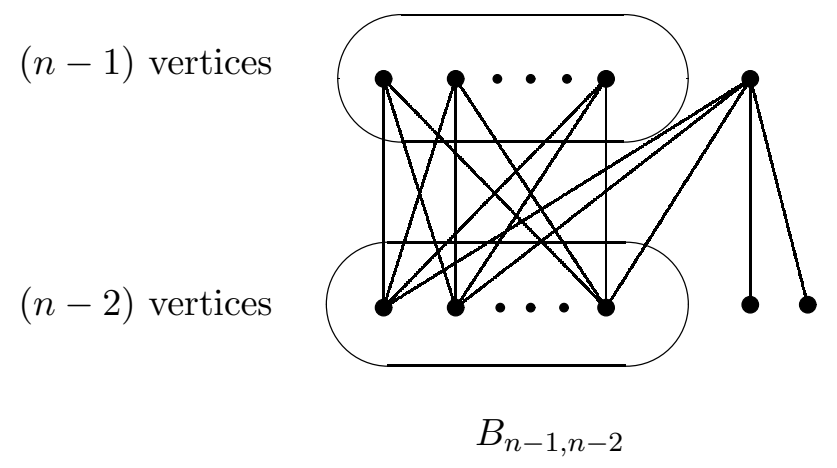

Figure 2: The extremal graph $B_{n-1, n-2}$ of Theorem 1.2 .

then write $2 G=G \cup H$ for short. Denote by $G \vee H$ the join of two graphs $G$ and $H$, which is the graph such that $V(G \vee H)=V(G) \cup V(H)$ and $E(G \vee H)=E(G) \cup E(G) \cup\{v u$ : $u \in V(G)$ and $v \in V(H)\}$. The graph $S_{n, k}$ is obtained from a copy of $K_{k}$ by adding $n-k$ vertices, each of which has neighborhood $V\left(K_{k}\right)$ i.e. $S_{n, k} \cong K_{k} \vee(n-k) K_{1}$. In the following, we first give a distance spectral radius condition which guarantees a graph to have a perfect matching.

Theorem 1.1. Let $G$ be a connected graph with order $n$ and $n \geq 4$ be an even integer.

(i) For $n \leq 10$, if $\lambda_{1}(D(G)) \leq \lambda_{1}\left(D\left(S_{n, \frac{n}{2}-1}\right)\right)$, then $G$ contains a perfect matching unless $G \cong S_{n, \frac{n}{2}-1}$.

(ii) For $n \geq 12$, if $\lambda_{1}(D(G)) \leq \lambda_{1}\left(D\left(G^{*}\right)\right)$, then $G$ contains a perfect matching unless

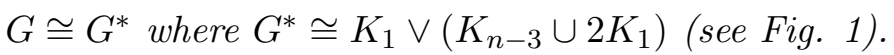

A connected bipartite graph with two parts of size $n_{1}$ and $n_{2}$. We say that it is balanced if $n_{1}=n_{2}$. Note that if a connected bipartite graph is not balanced. Then it has no perfect matching. Let $B_{n-1, n-2}$ be the graph obtained from $K_{n, n-2}$ by attaching two pendent vertices to a vertex in $n$-vertex part (see Fig. 2). Then we have the following result.

Theorem 1.2. Let $G$ be a connected balanced bipartite graph with order $2 n$ where $n$ is an 


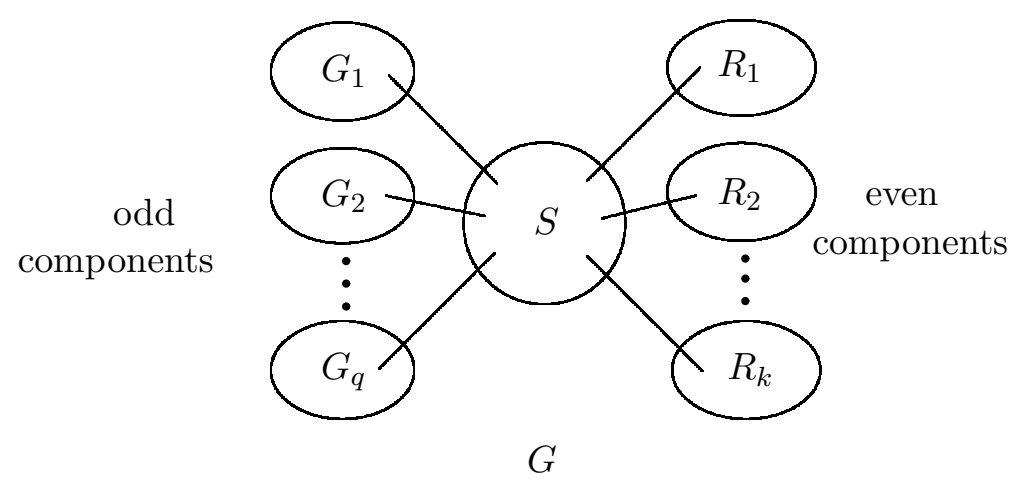

Figure 3: The graph in Tutte's theorem.

integer and $n \geq 3$. If $\lambda_{1}(D(G)) \leq \lambda_{1}\left(D\left(B_{n-1, n-2}\right)\right)$, then $G$ has a perfect matching unless $G \cong B_{n-1, n-2}$ (see Fig. Q 2 ).

\section{Proofs}

For $S \subseteq V(G)$, the subgraph induced by $V(G)-S$ is denoted by $G-S$. A component is called an odd (even) component if the number of vertices in this component is odd (even) and let $o(G)$ denote the number of odd components of $G$.

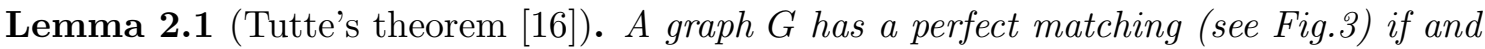
only if

$$
o(G-S) \leq|S| \text { for each } S \subseteq V(G) .
$$

Let $W(G)=\sum_{i<j} d_{i j}$ be the Wiener index of a connected graph $G$ with order $n$. Note that $\lambda_{1}(D(G))=\max _{\mathbf{x} \in \mathbb{R}^{n}} \frac{\mathbf{x}^{t} D(G) \mathbf{x}}{\mathbf{x}^{t} \mathbf{x}}$. Then we have

$$
\lambda_{1}(D(G))=\max _{\mathbf{x} \in \mathbb{R}^{n}} \frac{\mathbf{x}^{t} D(G) \mathbf{x}}{\mathbf{x}^{t} \mathbf{x}} \geq \frac{\mathbf{1}^{t} D \mathbf{1}}{\mathbf{1}^{t} \mathbf{1}} \geq \frac{2 W(G)}{n},
$$

where $\mathbf{1}=(1,1, \ldots, 1)^{t}$. Now we give the proof of Theorem 1.1 .

Proof of Theorem 1.1. By way of contradiction assume that $G$ has no perfect matching with the minimum distance spectral radius. By Lemma 2.1, there exists $S \subset V(G)$ such that $q-|S|>0$, where $o(G-S)=q$ and all components of $G-S$ are odd, otherwise, we can remove one vertex from each even component to the set $S$, in consequence, the number of odd component and the number of vertices in $S$ have the same increase, so that $q$ is always larger than $|S|$ and all components of $G-S$ are odd. Note that $G$ is connected, which implies that $S$ is not empty. $|S|$ and $q$ have the same parity because $n$ is even, so $q-|S| \geq 2$. Let $G^{\prime}$ be the graph obtained from $G$ by joining $S$ and $G-S$ and by adding 
edges in $S$ and in all components of $G-S$ so that all components of $G-S$ and $G[S]$ are cliques. It's clear that $G^{\prime} \cong K_{s} \vee\left(K_{n_{1}} \cup K_{n_{2}} \cup \cdots \cup K_{n_{q}}\right)$ where $|S|=s, n_{1} \geq n_{2} \geq \cdots \geq$ $n_{q} \geq 1$ and $n_{1}+n_{2}+\cdots+n_{q}=n-s$. Note that $G^{\prime} \cong G$, otherwise, referring to the Perron-Frobenius theorem, we have $\lambda_{1}\left(D\left(G^{\prime}\right)\right)<\lambda_{1}(D(G))$, a contradiction. Suppose $n_{2}=n_{3}=\cdots=n_{q}=1$, then we get a graph $G^{\prime \prime} \cong K_{s} \vee\left(K_{n-s-(q-1)} \cup(q-1) K_{1}\right)$.

Claim 1. $\lambda_{1}\left(D\left(G^{\prime}\right)\right) \geq \lambda_{1}\left(D\left(G^{\prime \prime}\right)\right)$ with equality if and only if $G^{\prime} \cong G^{\prime \prime}$.

If $n_{1}=1$, then $n_{1}=n_{2}=n_{3}=\cdots=n_{q}=1$ and $G^{\prime} \cong G^{\prime \prime}$. Now we consider $n_{1} \geq 3$. Denote the vertex set of $G^{\prime \prime}$ by $V\left(G^{\prime \prime}\right)=V\left(K_{s}\right) \cup V\left(K_{n-s-(q-1)}\right) \cup V\left((q-1) K_{1}\right)$. Suppose that $X$ is the Perron vector of $D\left(G^{\prime \prime}\right)$, and let $x(v)$ denote the entry of $X$ corresponding to the vertex $v \in V\left(G^{\prime \prime}\right)$. By symmetry, it is easy to see that all vertices of $V\left(K_{s}\right)$ (resp. $V\left(K_{n-s-(q-1)}\right)$ and $\left.V\left((q-1) K_{1}\right)\right)$ have the same entries in $X$. Thus we can suppose $x(u)=a$ for any $u \in V\left((q-1) K_{1}\right), x(v)=b$ for any $v \in V\left(K_{n-s-(q-1)}\right)$ and $x(w)=c$ for any $w \in V\left(K_{s}\right)$. Then

$$
\left\{\begin{array}{l}
\lambda_{1}\left(D\left(G^{\prime \prime}\right)\right) a=s c+2(n-s-q+1) b+2(q-2) a, \\
\lambda_{1}\left(D\left(G^{\prime \prime}\right)\right) b=s c+(n-s-q) b+2(q-1) a, \\
\lambda_{1}\left(D\left(G^{\prime \prime}\right)\right) c=(s-1) c+(n-s-q+1) b+(q-1) a .
\end{array}\right.
$$

Thus,

$$
a=\left[1+\frac{n-s-q}{\lambda_{1}\left(D\left(G^{\prime \prime}\right)\right)+2}\right] b .
$$

It follows that

$$
\begin{aligned}
& \lambda_{1}\left(D\left(G^{\prime}\right)\right)-\lambda_{1}\left(D\left(G^{\prime \prime}\right)\right) \\
\geq & X^{t}\left(D\left(G^{\prime}\right)-D\left(G^{\prime \prime}\right)\right) X \\
= & n_{1} \sum_{k=2}^{q}\left(n_{k}-1\right) b^{2}+\left(n_{2}-1\right)\left[\left(n-s-n_{2}-(q-2)\right) b^{2}-2 a b\right] \\
+ & \left(n_{3}-1\right)\left[\left(n-s-n_{3}-(q-2)\right) b^{2}-2 a b\right]+\left(n_{4}-1\right)\left[\left(n-s-n_{4}-(q-2)\right) b^{2}-2 a b\right] \\
+ & \cdots+\left(n_{q}-1\right)\left[\left(n-s-n_{q}-(q-2)\right) b^{2}-2 a b\right] .
\end{aligned}
$$

Since $n_{1} \geq 3$ and $n_{2} \geq n_{3} \geq \cdots \geq n_{q} \geq 1$, in order to show $\lambda_{1}\left(D\left(G^{\prime}\right)\right)-\lambda_{1}\left(D\left(G^{\prime \prime}\right)\right)>0$, we only need to prove $\left(n-s-n_{2}-(q-2)\right) b^{2}-2 a b>0$.

Note that $K_{n-q+1}$ is a subgraph of $G^{\prime \prime}$. Then $\lambda_{1}\left(D\left(G^{\prime \prime}\right)\right)>\lambda_{1}\left(D\left(K_{n-q+1}\right)\right)=n-q$, 
which implies

$$
\begin{aligned}
& \left(n-s-n_{2}-(q-2)\right) b^{2}-2 a b \\
= & b^{2}\left(n-s-n_{2}-q-\frac{2 n-2 s-2 q}{\lambda_{1}\left(D\left(G^{\prime \prime}\right)\right)+2}\right) \\
> & b^{2}\left(n-s-n_{2}-q-\frac{2 n-2 s-2 q}{n-q+2}\right) \\
= & b^{2}\left(n-s-n_{2}-q-2+\frac{2 s+4}{n-q+2}\right) \\
> & b^{2}\left(n-s-n_{2}-q-2\right) \\
= & b^{2}\left(n_{1}+n_{2}+\cdots+n_{q-1}+n_{q}-n_{2}-q-2\right) \\
= & b^{2}\left(\sum_{i=1, i \neq 2}^{q}\left(n_{i}-1\right)-1\right) \\
> & 0 .
\end{aligned}
$$

So Claim 1 holds.

So $G \cong G^{\prime \prime}$, or there will be a contradiction. We know that $q-|S| \geq 2$. Let $\widetilde{G} \cong$ $K_{s} \vee\left(K_{n-2 s-1} \cup(s+1) K_{1}\right)$. We compare the distance spectral radius of $G^{\prime \prime}$ and $\widetilde{G}$ in the following.

Claim 2. $\lambda_{1}\left(D\left(G^{\prime \prime}\right)\right) \geq \lambda_{1}(D(\widetilde{G}))$ with equality if and only if $G^{\prime \prime} \cong \widetilde{G}$.

Recall $G^{\prime \prime} \cong K_{s} \vee\left(K_{n-s-(q-1)} \cup(q-1) K_{1}\right)$. If $q=s+2$, then $\widetilde{G} \cong G^{\prime \prime}$. Now we consider $q \geq s+4$. Denote the vertex set of $\widetilde{G}$ by $V(\widetilde{G})=V\left(K_{s}\right) \cup V\left(K_{n-2 s-1}\right) \cup$ $V\left((s+1) K_{1}\right)$. Suppose that $Y$ is the Perron vector of $D(\widetilde{G})$, and let $Y(v)$ denote the entry of $Y$ corresponding to the vertex $v \in V(\widetilde{G})$. By symmetry, it is easy to see that all vertices of $V\left(K_{s}\right)$ (resp. $V\left(K_{n-2 s-1}\right)$ and $V\left((s+1) K_{1}\right)$ ) have the same entries in $X$. Thus we can suppose $Y(u)=y_{1}$ for any $u \in V\left((s+1) K_{1}\right), Y(v)=y_{2}$ for any $v \in V\left(K_{n-2 s-1}\right)$ and $Y(w)=y_{3}$ for any $w \in V\left(K_{s}\right)$. Let $n_{1}^{\prime}=n-s-(q-1) \geq 1$ and $G^{\prime \prime} \cong K_{s} \vee\left(K_{n_{1}^{\prime}} \cup(q-1) K_{1}\right)$. Then

$$
\begin{aligned}
& \lambda_{1}\left(D\left(G^{\prime \prime}\right)\right)-\lambda_{1}(D(\widetilde{G})) \\
\geq & Y^{t}\left(D\left(G^{\prime \prime}\right)-D(\widetilde{G})\right) Y \\
= & n_{1}^{\prime}(q-s-2) y_{2}^{2}+\left(n_{1}^{\prime}+q-s-3\right)(q-s-2) y_{2}^{2} \\
= & y_{2}^{2}\left[q^{2}+\left(2 n_{1}^{\prime}-2 s-5\right) q+s^{2}+5 s-2 n_{1}^{\prime} s-4 n_{1}^{\prime}+6\right] .
\end{aligned}
$$


Since

$$
\frac{-\left(2 n_{1}^{\prime}-2 s-5\right)}{2}=-n_{1}^{\prime}+s+\frac{5}{2}<s+4
$$

we obtain

$$
\begin{aligned}
& y_{2}^{2}\left[q^{2}+\left(2 n_{1}^{\prime}-2 s-5\right) q+s^{2}+5 s-2 n_{1}^{\prime} s-4 n_{1}^{\prime}+6\right] \\
\geq & y_{2}^{2}\left[(s+4)^{2}+\left(2 n_{1}^{\prime}-2 s-5\right)(s+4)+s^{2}+5 s-2 n_{1}^{\prime} s-4 n_{1}^{\prime}+6\right] \\
= & y_{2}^{2}\left(4 n_{1}^{\prime}+2\right) \\
> & 0 .
\end{aligned}
$$

So Claim 2 holds.

So $G \cong \widetilde{G}$, or there will be a contradiction. Let $G^{*} \cong K_{1} \vee\left(K_{n-3} \cup 2 K_{1}\right)$. In the end, we shall show that $G^{*}$ contains the minimum distance spectral radius and no perfect matching under the most situations.

Claim 3. If $n \geq 2 s+4$, then $\lambda_{1}(D(\widetilde{G})) \geq \lambda_{1}\left(D\left(G^{*}\right)\right)$ with equality if and only if $\widetilde{G} \cong G^{*}$.

Recall $\widetilde{G} \cong K_{s} \vee\left(K_{n-2 s-1} \cup(s+1) K_{1}\right)$. If $s=1$, then $G^{*} \cong \widetilde{G}$. Now we suppose $s \geq 2$ so that $n \geq 2 s+4 \geq 8$. Then the quotient matrix of the partition $\left\{V\left(K_{n-2 s-1}, V\left(K_{s}\right), V((s+\right.\right.$ 1) $\left.\left.\left.K_{1}\right)\right)\right\}$ of $\widetilde{G}$ is

$$
\left(\begin{array}{ccc}
n-2 s-2 & s & 2(s+1) \\
n-2 s-1 & s-1 & s+1 \\
2(n-2 s-1) & s & 2 s
\end{array}\right)
$$

the characteristic polynomial of the matrix is

$f(x)=x^{3}-(s+n-3) x^{2}-\left(2 s n-5 s^{2}+5 n-6 s-6\right) x+n s^{2}-2 s^{3}-s n+2 s^{2}-4 n+6 s+4$.

We know that $\lambda_{1}(D(\widetilde{G}))$ is the largest root of $f(x)=0$. Since $\lambda_{1}\left(D\left(G^{*}\right)\right)=\theta(n)$ (simply $\theta$ ) is the largest root of the equation $q(x)=x^{3}-(n-2) x^{2}-(7 n-17) x-4 n+10=0$, we have

$$
\begin{aligned}
h(\theta) & =f(\theta)-q(\theta) \\
& =-(s-1) \theta^{2}-\left(2 n s-5 s^{2}-2 n-6 s+11\right) \theta+n s^{2}-2 s^{3}-s n+2 s^{2}+6 s-6 \\
& =(s-1)\left(-\theta^{2}+(-2 n+5 s+11) \theta+s n-2 s^{2}+6\right) .
\end{aligned}
$$


Moreover, $\theta=\lambda_{1}\left(D\left(G^{*}\right)\right) \geq \frac{2 W\left(G^{*}\right)}{n}=\frac{n^{2}+3 n-10}{n} \geq n+1$ and $s \geq 2$. To show $\lambda_{1}(D(\widetilde{G}))>$ $\lambda_{1}\left(D\left(G^{*}\right)\right)$, we only need to prove $h_{1}(\theta)=-\theta^{2}+(-2 n+5 s+11) \theta+s n-2 s^{2}+6<0$ when $\theta \geq n+1$, then $h(\theta)<h_{1}(\theta)<0$. Note that

$$
\frac{-2 n+5 s+11}{2}=-n+\frac{5}{2} s+\frac{11}{2}<n+1 .
$$

So when $\theta \geq n+1, h_{1}(\theta)$ monotonically decreases as $\theta$ increases and $h_{1}(\theta) \leq h_{1}(n+1)$. Let $g(n)=h_{1}(n+1)=-3 n^{2}+(6 s+7) n-2 s^{2}+5 s+16$ where $n \geq 2 s+4$. Then

$$
\frac{6 s+7}{6}=s+\frac{7}{6}<2 s+4
$$

So when $n \geq 2 s+4, g(n)$ is monotonically decreasing and $h(\theta)<h_{1}(\theta) \leq g(n) \leq$ $g(2 s+4)=-2 s^{2}-5 s-4<0$. So Claim 3 holds.

If $n=2 s+2$, observe that $\widetilde{G} \cong S_{n, \frac{n}{2}-1}$ and the quotient matrix of the partition $\left\{V\left(K_{\frac{n}{2}-1}\right), V\left(\left(\frac{n}{2}+1\right) K_{1}\right)\right\}$ of $S_{n, \frac{n}{2}-1}$ is

$$
\left(\begin{array}{cc}
\frac{n}{2}-2 & \frac{n}{2}+1 \\
\frac{n}{2}-1 & n
\end{array}\right) .
$$

By a simple calculation,

$$
\lambda_{1}\left(D\left(S_{n, \frac{n}{2}-1}\right)\right)=\frac{3 n-4+\sqrt{n^{2}+24 n-16}}{4} .
$$

Claim 4. If $n=2 s+2$ and $n \geq 12$, then $\lambda_{1}\left(D\left(S_{n, \frac{n}{2}-1}\right)\right)>\lambda_{1}\left(D\left(G^{*}\right)\right)$.

Let $n=2 s+2$, then $g(n)<0$ when $n \geq 12$. So Claim 4 holds.

Claim 5. If $n=2 s+2$ and $n \leq 10$, then $\lambda_{1}\left(D\left(S_{n, \frac{n}{2}-1}\right)\right) \leq \lambda_{1}\left(D\left(G^{*}\right)\right)$ with equality if and only if $G^{*} \cong S_{n, \frac{n}{2}-1}$.

If $s=1$ and $n=4$, then $S_{n, \frac{n}{2}-1} \cong G^{*}$. If $s=2$ and $n=6$, then $\lambda_{1}\left(D\left(S_{6,2}\right)\right)=\frac{7+\sqrt{41}}{2}$ and $q\left(\frac{7+\sqrt{41}}{2}\right)<0$, so $\lambda_{1}\left(D\left(S_{6,2}\right)\right)<\theta(6)$. If $s=3$ and $n=8$, then $\lambda_{1}\left(D\left(S_{8,3}\right)\right)=5+\sqrt{15}$ and $q(5+\sqrt{15})<0$, so $\lambda_{1}\left(D\left(S_{8,3}\right)\right)<\theta(8)$. If $s=4$ and $n=10$, then $\lambda_{1}\left(D\left(S_{10,4}\right)\right)=11$ and $q(11)<0$, so $\lambda_{1}\left(D\left(S_{10,4}\right)\right)<\theta(10)$. So Claim 5 holds.

In conclusion, $G \cong G^{*}$ if $n \geq 12$ and $G \cong S_{n, \frac{n}{2}-1}$ if $4 \leq n \leq 10$, otherwise $G$ doesn't contain the minimum distance spectral radius, a contradiction.

In some specific applications, one needs to find a matching in a bipartite graph which can cover one partite. Essential and sufficient conditions for the existence of such a matching was first proposed by Hall [7]. Let $S$ be a set of vertices in a graph $G$. The set of all neighbours of the vertices in $S$ is denoted by $N(S)$. 


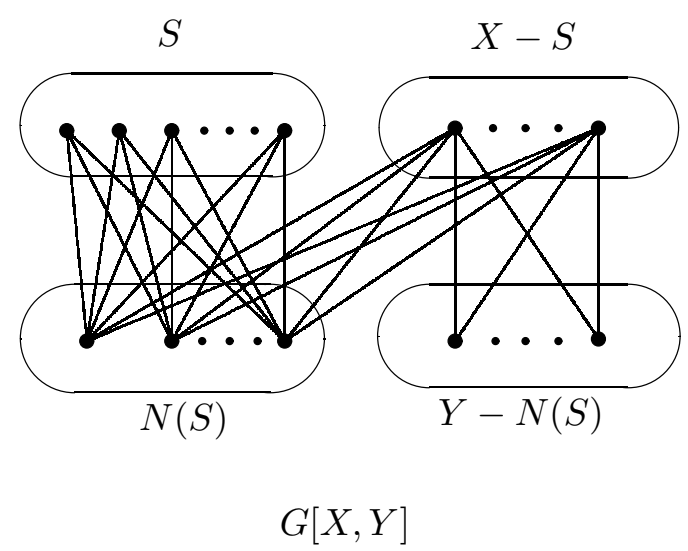

Figure 4: The graph $B_{|S|,|N(S)|}$ in Theorem 1.2 .

Lemma 2.2 (Hall's theorem [7]). A bipartite graph $G:=G[X, Y]$ has a matching which covers every vertex in $X$ if and only if

$$
|N(S)| \geq|S| \text { for each } S \subseteq X .
$$

For two vertex sets $X$ and $Y$, let $e(X, Y)$ be the set of all edges between $X$ and $Y$. Now we are in a position to prove Theorem 1.2 .

Proof of Theorem 1.2. Assume to the contrary that $G$ has no perfect matching with the minimum distance spectral radius. Let $G:=G[X, Y]$ be a connected balanced bipartite graph, where $|X|=|Y|=n$. By Lemma 1.2, since $G$ has no perfect matching, there exists $S \subset X$ and $|N(S)|<|S|$. Notice that there exists no edges between $S$ and $Y-N(S)$, otherwise, we can find a vertex $v \in S$ and $Y-N(S)$ contains its neighbors, a contradiction. Let $B_{s, p}$ be the connected balanced bipartite graph obtained from $G$ by joining $S$ and $N(S)$, $X-S$ and $Y-N(S)$ and by adding all possible edges between $X-S$ and $N(S)$ where $|S|=s,|N(S)|=p$ and $1 \leq p<s \leq n-1$, so that $B_{s, p} \cong K_{s, p} \cup K_{n-s, n-p}+e(N(S), X-S)$,

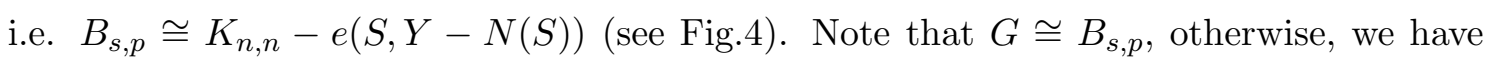
$\lambda_{1}\left(D\left(B_{s, p}\right)\right)<\lambda_{1}(D(G))$ by Perron-Frobenius theorem, a contradiction.

Claim 1. $\lambda_{1}\left(D\left(B_{s, p}\right)\right) \geq \lambda_{1}\left(D\left(B_{s, s-1}\right)\right)$ with equality if and only if $B_{s, p} \cong B_{s, s-1}$.

If $p=s-1$, then $B_{s, p} \cong B_{s, s-1}$. Now we consider $1 \leq p \leq s-2$. Denote the vertex set of $B_{s, s-1}$ by $V\left(B_{s, s-1}\right)=S \cup(X-S) \cup N(S) \cup(Y-N(S))$ where $|S|=s$ and $|N(S)|=s-1$. Referring to the Perron-Frobenius theorem, suppose that a positive vector $Z$ is the Perron vector of $D\left(B_{s, s-1}\right)$, and let $Z(v)$ denote the entry of $Z$ corresponding to the vertex $v \in V\left(B_{s, s-1}\right)$. By symmetry, it is easy to see that all vertices of $S$ (resp. $X-S, N(S)$ and $Y-N(S))$ have the same entries in $Z$. Thus we can suppose $Z(u)=z_{1}$ 
for any $u \in S, Z(v)=z_{2}$ for any $v \in X-S, Z(w)=z_{3}$ for any $w \in N(S)$ and $Z(z)=z_{4}$ for any $z \in Y-N(S)$. Therefore, we have

$$
\begin{aligned}
& \lambda_{1}\left(D\left(B_{s, p}\right)\right)-\lambda_{1}\left(D\left(B_{s, s-1}\right)\right) \\
\geq & Z^{t}\left(D\left(B_{s, p}\right)-D\left(B_{s, s-1}\right)\right) Z \\
= & 4 s(s-1-p) z_{1} z_{3} \\
> & 0 .
\end{aligned}
$$

So Claim 1 holds.

Therefore, $G \cong B_{s, s-1}$, or there will be a contradiction.

Claim 2. $\lambda_{1}\left(D\left(B_{s, s-1}\right)\right) \geq \lambda_{1}\left(D\left(B_{n-1, n-2}\right)\right)$ with equality if and only if $B_{s, s-1} \cong B_{n-1, n-2}$.

If $s=n-1$, then $B_{s, s-1} \cong B_{n-1, n-2}$. Now consider $2 \leq s \leq n-2$. The quotient matrix of the partition $\{S, X-S, N(S), Y-N(S)\}$ of $B_{s, s-1}$ where $|S|=s \leq n-2$ and $|N(S)|=s-1$ is

$$
\left(\begin{array}{cccc}
2 s-2 & s-1 & 2 n-2 s & 3 n-3 s+3 \\
s & 2 s-4 & n-s & 2 n-2 s+2 \\
2 s & s-1 & 2 n-2 s-2 & n-s+1 \\
3 s & 2 s-2 & n-s & 2 n-2 s
\end{array}\right)
$$

the characteristic polynomial of the matrix is

$$
\begin{aligned}
f(\lambda)=\lambda^{4} & +(-4 n+8) \lambda^{3}+\left(3 n^{2}-8 n s+8 s^{2}-24 n-8 s+24\right) \lambda^{2} \\
& +\left(8 n^{2} s-8 n s^{2}+12 n^{2}-32 n s+40 s^{2}-48 n-40 s+32\right) \lambda \\
& -12 n^{2} s^{2}+24 s^{3} n-12 s^{4}+28 n^{2} s-52 n s^{2}+24 s^{3}+12 n^{2} \\
& -20 s n+36 s^{2}-32 n-48 s+16 .
\end{aligned}
$$

We know that $\lambda_{1}\left(D\left(B_{s, s-1}\right)\right)$ is the largest root of $f(\lambda)=0$. Since $\lambda_{1}\left(D\left(B_{n-1, n-2}\right)\right)$, written by $\rho$, is the largest root of the equation

$q(\lambda)=\lambda^{4}+(-4 n+8) \lambda^{3}+\left(3 n^{2}-40 n+40\right) \lambda^{2}+\left(28 n^{2}-144 n+112\right) \lambda+20 n^{2}-88 n+64=0$, 
we have

$$
\begin{aligned}
h(\rho) & =f(\rho)-q(\rho) \\
& =\left(8 s^{2}-(8 n+8) s+16 n-16\right) \rho^{2} \\
& +\left(8 s n^{2}-8 n s^{2}-16 n^{2}-32 n s+40 s^{2}+96 n-40 s-80\right) \rho \\
& -12 n^{2} s^{2}+24 s^{3} n-12 s^{4}+28 s n^{2}-52 n s^{2}+24 s^{3}-8 n^{2}-20 s n+36 s^{2}+56 n-48 s-48 .
\end{aligned}
$$

Now we need to state $h(\rho)<0$. Firstly, we give a lower bound on $\rho$,

$$
\frac{2 W\left(B_{n-1, n-2}\right)}{2 n}=2 n+5-\frac{6}{n}<\rho .
$$

By a simple computation, we obtain

$$
8 s^{2}-(8 n+8) s+16 n-16 \leq 0 \text { for } 2 \leq s \leq n-2,
$$

and

$$
\frac{8 s n^{2}-8 n s^{2}-16 n^{2}-32 n s+40 s^{2}+96 n-40 s-80}{-2\left(8 s^{2}-(8 n+8) s+16 n-16\right)}=\frac{n-5}{2}<2 n<\rho .
$$

Thus, $h(\rho)$ is monotonically decreasing for $\rho>2 n$ and $h(\rho)<h(2 n)$. Let $h(2 n)$ be written by $g(s)$ and we only need to prove $g(s)<0$ for $2 \leq s \leq n-2$ in the following steps. Based on Matlab programming, we have

$$
g(s)=-4(s-2)(-s+n-1)\left[-3 s^{2}+(3 s+3) n+19 n+4 n^{2}+6\right] .
$$

It is easy to see $s-2 \geq 0$ and $-s+n-1>0$. Define $r(s)=-3 s^{2}+(3 s+3) n+19 n+4 n^{2}+6$, we can easily get for $2 \leq s \leq n-2$ and $n \geq 3$,

$$
\begin{aligned}
& r(s)=-3 s^{2}+(3 s+3) n+19 n+4 n^{2}+6 \\
> & \min \{r(2), r(n-2)\} \\
= & \min \left\{4 n^{2}+25 n, 4 n^{2}+28 n-12\right\} \\
> & 0 .
\end{aligned}
$$

Therefore, $h(\rho)<h(2 n)=g(s)<0$. So Claim 2 holds.

In conclusion, $G \cong B_{n-1, n-2}$ has the minimum distance spectral radius among all $2 n$-vertex balanced bipartite graphs without a perfect matching. 


\section{References}

[1] M. Aouchiche, P. Hansen, Proximity, remoteness and distance eigenvalues of a graph, Discrete Appl. Math. 222213 (2016) 17-25.

[2] M. Aouchiche, P. Hansen, Distance spectra of graphs: A survey, Linear Algebra Appl. 458 (2014) 301-386.

[3] A. E. Brouwer, W. H. Haemers, Eigenvalues and perfect matchings, Linear Algebra Appl. 395 (2005) 155-162.

[4] A. Chang, Bounds on the second largest eigenvalue of a tree with perfect matchings, Linear Algebra Appl. 283 (1998) 247-255.

[5] L. Feng, G. Yu, X. Zhang, Spectral radius of graphs with given matching number, Linear Algebra Appl. 422 (2007) 133-138.

[6] R.L. Graham, H.O. Pollack, On the addressing problem for loop switching, Bell Syst. Tech. J. 50 (1971) 2495-2519.

[7] P. Hall, On a Theorem of Frobenius, Proc. London Math. Soc. 40 (2) (1936) 468-501.

[8] Y. Hou, J. Li, Bounds on the largest eigenvalues of trees with a given size of matching, Linear Algebra Appl. 342 (2002) 203-217.

[9] A. Ilić, Distance spectral radius of trees with given matching number, Discret. Appl. Math. 158 (2010) 1799-1806.

[10] H. Lin, On the sum of $k$ largest distance eigenvalues of graphs, Discrete Appl. Math. 259 (2019) 153-159.

[11] H. Lin, Y. Zhang, Extremal problems on distance spectra of graphs, Discrete Applied Math. 289 (2021) 139-147.

[12] Z. Liu, On spectral radius of the distance matrix, Appl. Anal. Discret. Math. 4 (2010) 269-277.

[13] Z. Lou, H. Lin, Distance eigenvalues of a cograph and their multiplicities, Linear Algebra Appl. 608 (2021) 1-12. 
[14] H. Lu, J. Luo, Extremal unicyclic graphs with minimal distance spectral radius, Discuss. Math. Graph Theory 34 (2014) 735-749.

[15] S. O, Spectral radius and matchings in graphs, Linear Algebra Appl. (2020) https://doi.org/10.1016/j.laa.2020.06.004.

[16] W.T. Tutte, The factorization of linear graphs, J. Lond. Math. Soc. 22 (1947) 107111.

[17] J.Xue, H. Lin, J. Shu, On the second largest distance eigenvalue of a block graph, Linear Algebra Appl. 591 (2020) 284-298.

[18] M. Zhang, S. Li, Extremal cacti of given matching number with respect to the distance spectral radius, Applied Math. Comput. 291 (2016) 89-97.

[19] X. Zhang, On the distance spectral radius of unicyclic graphs with perfect matching, Electron. J. Linear Algebra 27 (2014) 569-587. 